\title{
Neuronal Fiber Connections based on A*-Pathfinding
}

\author{
D. Merhof ${ }^{a, b}$, F. Enders ${ }^{a, b}$, P. Hastreiter ${ }^{a, b}$, O. Ganslandt ${ }^{c}$, \\ R. Fahlbusch ${ }^{c}$, C. Nimsky ${ }^{c}$ and M. Stamminger ${ }^{b}$ \\ ${ }^{a}$ Neurocenter, Dept. of Neurosurgery, University of Erlangen-Nuremberg, Germany \\ ${ }^{b}$ Computer Graphics Group, University of Erlangen-Nuremberg, Germany \\ ${ }^{c}$ Dept. of Neurosurgery, University of Erlangen-Nuremberg, Germany
}

\begin{abstract}
Diffusion tensor imaging has shown potential in providing information about the location of white matter tracts within the human brain. Based on this data, a novel approach is presented establishing connectivity between functional regions using pathfinding. The probability distribution function of the local tensor thereby controls the state space search performed by pathfinding. Additionally, it serves as an indicator for the reliability of the computed paths visualized by color encoding. Besides the capability to handle noisy data, the probabilistic nature of the approach is also able to cope with crossing or branching fibers. The algorithm thus guarantees to establish a connection between cortical regions and on the same hand provides information about the probability of the obtained connection. This approach is especially useful for investigating the connectivity between certain centers of the brain as demonstrated by reconstructed connections between motor and sensory speech areas.
\end{abstract}

Keywords: Diffusion Tensor Imaging, functional MRI, Pathfinding

\section{INTRODUCTION}

Understanding the function and structure of the human brain is an area of great interest and ongoing research in neuroscience. In recent years, medical imaging techniques such as functional MRI (fMRI) and diffusion tensor imaging (DTI) have emerged providing valuable information for this purpose.

fMRI enables the localization of active brain areas such as motor and sensory speech areas. DTI measures diffusion of water which is anisotropic in tissue with a high degree of directional organization such as white matter. Thereby, information about the location and connectivity of neuronal fibers is provided.

The combination of white matter connectivity and localized cortical areas is of high interest for a deeper understanding of the functionality of the human brain. Regarding the motor and sensory speech areas, the Broca's and Wernicke's areas located at the cerebral cortex, it is generally accepted that these areas are functionally related in speech processing. A better insight concerning their functional relationship is of high interest for neuroscience.

The reconstruction of white matter tracts from DTI data is commonly solved by tracking algorithms. However, fiber tracking has several restrictions that prevent it from being appropriate for connecting cortical areas. Tracking initialized from one speech area is not guaranteed to find the second speech area at all. The reason is that fiber tracking is not goal oriented since regions of interest (ROIs) used in fiber tracking just serve as seed regions or to select fibers running through the ROI. Additionally, imaging noise, integration errors and partial volume effects may cause the fibers to miss the goal. A further aspect is that fiber tracking algorithms are only able to progress as far as the certainty of the fiber direction represented by a strong anisotropy is sufficiently high. Near subcortical or cortical regions this effect forces the fiber propagation to terminate.

These problems have been partly addressed by several groups. Filtering techniques ${ }^{1}$ as well as approaches that aim at reconstructing the true tensor matrix on each voxel ${ }^{2}$ enable to handle imaging noise. Another class of 
algorithms tries to circumvent partial volume effects by incorporating the probability distribution function of the tensor into tracking resulting in probabilistic or regularization approaches. ${ }^{3,4}$ Other probabilistic approaches consider arrival times of diffusion fronts ${ }^{5}$ or concentration distributions. ${ }^{6}$ The connectivity problem has also been investigated using standard fiber tracking by employing seed regions including both grey matter and adjoining white matter. ${ }^{7}$

In this paper, a novel method is presented that establishes a connection between brain areas using pathfinding while overcoming the challenges mentioned above. As a result, connections between two predefined areas are calculated including the related probability. Pathfinding is thereby applied to generate a connection between the input regions. In artificial intelligence (AI), pathfinding algorithms are commonly used for solving problems associated with such a state space search. These highly efficient algorithms are thereby applied to derive a solution or path that connects an initial and final state corresponding to functional brain regions.

The approach is thereby locally guided by cost functions based on the probability distribution function of each tensor. In this way, uncertainty in path direction is modeled controlling the pathfinding procedure. Relative probabilities of different directions are taken into account which guarantees the propagation of the path even if a voxel with low probability is encountered. The approach thus naturally handles imaging noise as well as branching, crossing or kissing fibers. The probabilistic nature of the cost functions prevents the algorithm from drifting to errant routes. Additionally, the probabilities of each step are recorded and visualized providing a connectivity estimate for the whole path.

\section{METHOD}

This section provides a general introduction to pathfinding (Section 2.1) and continues with a short outline of the $\mathrm{A}^{*}$ algorithm (Section 2.2) which was adapted for establishing a connection between cortical areas. The pathfinding procedure highly depends on appropriate thresholds (Section 2.3), cost functions (Section 2.4) and heuristics (Section 2.5).

\subsection{Pathfinding}

Pathfinding is a search algorithm performing state space search to compute a path with lowest cost between two points. $^{8}$ Starting with an initial state or node, the algorithm builds up a graph consisting of weighted edges leading to successor states or nodes representing valid steps in solving the search problem. Reliability of a certain step is indicated by costs accumulated up to the current state and estimates about the remaining cost denoted as heuristics. Graph searching expands until a state or node is reached that is specified as a solution.

An extremely efficient pathfinding algorithm is $\mathrm{A}^{* 9,10}$ which is used in a variety of contexts in AI. It computes the path with lowest cost between an initial node and a goal node in an edge weighted graph. It employs a cost function and a heuristic to select appropriate successor states. Provided that the heuristic is good, A* is very fast and also finds the best possible solution. ${ }^{11}$

\section{2. $\mathrm{A}^{*}$ algorithm}

$\mathrm{A}^{*}$ works on a search area which in our case is the diffusion tensor dataset. The center points of the search area voxels are denoted as 'nodes'. The status of a node may be walkable or unwalkable according to a domain restriction threshold (Section 2.3). The search is initialized with a start node and a goal node. Beginning with the start node, in each step the neighbor nodes are determined and further processed based on thresholds and costs. To find the path with lowest cost, the searching process builds up a graph by searching outwards until the goal node is found.

For this purpose, the algorithm maintains two lists: An open list encompassing all nodes that need to be further considered and a closed list of nodes that have already been processed. When starting the search, the open list just contains the start node and the closed list is empty. The start node is taken from the open list and all walkable neighbor nodes are determined and inserted into the open list. Each node maintains a reference pointing to its parent node which is finally necessary to trace the path from the goal back to the start node. For each neighbor node on the open list the start node is thus saved as parent node. 
A crucial role for determining the best path are cost functions that provide a bias towards neighbor nodes that are more likely to lie on this path. The key equation for computing the cost to proceed to neighbor node $n$ is $F(n)=G(n)+H(n)$. Thereby, $F(n)$ is the accumulated cost which is considered for choosing an appropriate neighbor node in the graph. It is composed of the movement cost $G(n)$ and the heuristic $H(n)$. $G(n)$ is the movement cost accumulated for each single step following the path from the starting node to the current node. The heuristic $H(n)$ provides an estimate of the cost for reaching the goal node from node $n$.

The path with lowest cost is generated by repeatedly selecting the node with lowest cost from the open list, adding all walkable neighbors with assigned costs and a reference to the parent node to the open list and moving the selected node to the closed list. This process is repeated until the goal node is added to the open list and the path with the lowest cost is found. An outline of the algorithm is found in Figure 1.



Figure 1. Schematic outline of the $\mathrm{A}^{*}$ algorithm. For each square in the grid the shape of the tensor ellipsoid is indicated. Additionally, squares that have a low FA value and that are not walkable are denoted by a dark background. An example of fiber tracking (blue) as well as possible pathfinding results (green, red) are shown. Contrary to the fiber, pathfinding takes a slight turn in the end to reach the goal. Depending on the FA threshold and the heuristic, the red alternative may be taken. If the pathfinding algorithm is forced to take turns not in accordance with the local tensor shape, the probability of the whole path is low.

\subsection{Thresholding}

A possible connection between areas within the brain must be restricted to regions of white matter. The search domain is therefore limited to regions with anisotropic diffusion preventing the algorithm from expanding to regions that are definitely beyond white matter. Due to the restricted search area the size of the edge weighted graph is considerably reduced. Computation times become faster since less possible directions have to be processed and the graph expands more restrictively.

For the classification of white matter based on diffusion tensors a number of diffusion metrics such as fractional anisotropy (FA) or the apparent diffusion coefficient (ADC) exist. These metrics measure the degree of anisotropy which is generally agreed to be a characteristic property of white matter. Strongly aligned tissue such as white matter causes anisotropic diffusion resulting in linear diffusion tensors. In the case of crossing or kissing fibers, planar diffusion tensors are observed due to the partial volume effect. Contrarily, in areas of grey matter spherical tensors are mainly encountered. Among the available measures for anisotropy, we selected FA for domain restriction which is also a common fiber propagation threshold for fiber tracking algorithms.

In the context of pathfinding such a threshold is known as walkability threshold since nodes are thereby classified as possible successor nodes or forbidden nodes excluded from further consideration. 


\subsection{Cost function}

The cost function is an integral part of the algorithm. Depending on the cost function and the heuristic the direction to expand the graph is determined. For investigating the connectivity between brain areas the local diffusion tensor properties have to control the cost function:

Tensor shape: The unconditional probability of a fiber following a certain direction is directly given by the probability distribution function described by the tensor. The probability profile of the local tensor can be visualized by plotting the probability as a function of the azimuthal and polar angle of the corresponding ellipsoid. The ellipsoid is thereby scaled in such a way that the longest half axis is normalized to obtain a probability between 0 and 1 . Accordingly, the resulting surface describes the probability as a function of direction (Figure 2).

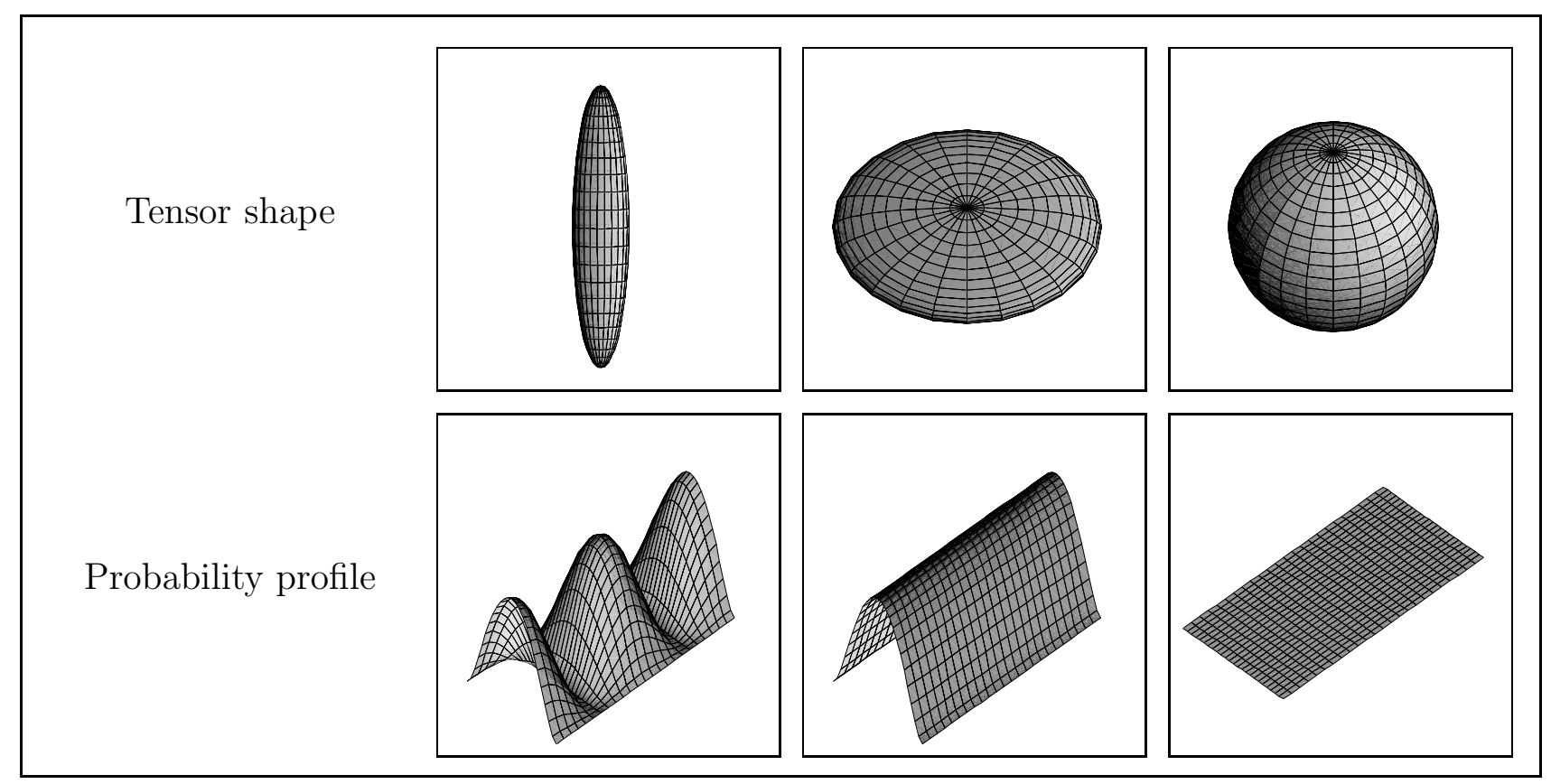

Figure 2. Linear, planar and spherical diffusion tensor ellipsoids (upper row) and corresponding probability distribution functions representing probability as a function of the two spherical angles $\Theta$ and $\phi$ (lower row)

Bending energy: This cost factor adds an increasing cost depending on the bending of the fiber. Intuitively, the bending energy and therefore the assigned cost takes its minimum if incoming and outgoing direction are the same. The bending cost is adjusted based on fiber modeling assuming the fibers to be somewhat stiff. A maximum probability and minimum cost is assigned if incoming and outgoing direction are equal, for a wider angle the probability decreases which implies a growing cost. A shifted and scaled cosine function is used to generate probability values between 0 and 1 . The cosine function was selected for several reasons: The profile of the function ensures that a small deviation from the incoming direction only causes a slightly lower probability whereas for higher deviations the gradient of the function decreases very fast assigning low probabilities. Additionally, the cosine just needs a single dot product and is therefore fast to compute.

Fractional anisotropy (FA): In addition to the walkability threshold based on fractional anisotropy, this quantity is also used to assign costs. The idea is to penalize paths that cross voxels with lower FA. 


\subsection{Heuristic}

The heuristic $H$ is an estimating function of the minimum cost from any voxel to the goal voxel and ensures that the search is oriented towards the goal voxel. The heuristic should be an as high as possible lower bound of the real cost which is unknown. If the heuristic overestimates the real cost, it is called inadmissible. In this case, it is not guaranteed that the algorithm finds the path with lowest cost but runs faster. If the cost for moving to the goal voxel $H$ is underestimated, then $\mathrm{A}^{*}$ is guaranteed to find the path with lowest cost. However, the lower the values of $H$, the more the algorithm expands and the slower it performs.

$H$ can be estimated in a variety of ways. The standard heuristic for grids is the Manhattan method. ${ }^{10}$ It computes the sum of voxels moved in $x, y$ and $z$ direction to reach the goal voxel $g$ from the current voxel $n$. Diagonal movement as well as unwalkable areas are not further considered. If the goal consists of more than one voxel, the minimum cost computed by the Manhattan method to any of the goal voxels is taken as heuristic.

$$
H(n, g)=\left\|\left(x_{n}-x_{g}\right)\right\|+\left\|\left(y_{n}-y_{g}\right)\right\|+\left\|\left(z_{n}-z_{g}\right)\right\|
$$

The heuristic aims at estimating the remaining distance between the current voxel and the goal which is usually farther. The closer the heuristic estimates the actual remaining distance, the the faster the algorithm will perform. However, if the distance is overestimated, there is no guarantee that the algorithm will compute the most possible path (the heuristic is called 'inadmissible').

Strictly speaking, in our datasets the Manhattan method is inadmissible since the remaining distance is slightly overestimated. It is used nevertheless since it its fast to compute and the overestimate is only minor. On the rare occasion that the resulting path is not the most possible, it will be nearly as possible.

If the goal consists of more than one voxel, the minimum cost computed by the Manhattan method to any of the goal voxels is taken as heuristic.

\section{RESULTS AND DISCUSSION}

To evaluate the algorithm we performed a number of experiments on two proband DTI datasets $(128 \times 128 \times 60$ voxels, voxel size: $1.875 \times 1.875 \times 1.9 \mathrm{~mm}^{3}$ ) acquired with a Siemens Sonata 1.5 Tesla scanner. For both datasets manually defined start and goal regions were used. Additionally, for the second dataset seed regions derived from fMRI were available. The paths derived by the algorithm were color encoded according to the probability of each segment assigning green color to a high, red color to low probability. In each experiment we varied the weights relating the components of the cost function resulting in different paths to obtain a more comprehensive representation. The following experiments were performed on the data:

(a) Comparison with fiber tracking: In a first step, conventional fiber tracking between two seed regions of a common tract was performed. The outcome of fiber tracking and pathfinding was compared and showed high correlation. This was verified for several tract systems of the human brain, namely the pyramidal tract and for parts of the corpus callosum.

(b) Probable connection between seed regions in same tract: In a second experiment the start and goal region were slightly shifted so that fiber tracking no longer produced fibers running through both seed regions. However, with pathfinding a connection was established showing high probability indicated in green in each section of the path (Figure 3, left). This was also verified for different tract systems.

(c) Less plausible connection: The next experiment provides the pathfinding algorithm with two distant areas located each in a different hemisphere of the brain. Pathfinding computes the most probable connection between these areas. However, parts of the resulting path (Figure 3, right) have a very low possibility denoted in red, which indicates that the connection as a whole is rather improbable. It has to be noticed that the algorithm provides the most probable connection based on the local tensor information, to judge wether the connection as a whole is feasible from the medical point of view has to be left to the clinician. 



Figure 3. Connections between manually defined regions displayed with FA data. Left: Fibers displayed in blue originating in the lower seed region just miss the upper one whereas pathfinding computes a very probable connection indicated in green. Right: The most probable connection between distant regions is shown, however the red segments indicate that a path between these areas is rather improbable.

(d) Connection between speech areas: The last experiment was performed using the proband dataset with functional speech areas as seed regions (Figure 4). The approach generated an arched connection between Broca's and Wernicke's area which is conform to medical knowledge. Since the derived paths are located within the gyri of the brain and do not cross a sulci which would indicate a wrong course, it may be anticipated that the underlying white matter structure is resembled correctly. With conventional fiber tracking this connection could not be reproduced, even if a very low FA threshold was applied.

All experiments were performed on a PC equipped with an Intel Pentium 4 (2.4 GHz). Computation times for a single path for the different setups were in the range of seconds up a few minutes (in all cases less than $5 \mathrm{~min}$ ) which highly depends on the choice of parameters and the start and goal region. More precisely, a reduced weight of the heuristic $H$ has the effect that the search space expands and path computation gets more expensive. Similarly, the longer the path the algorithm has to generate to reach the goal region, the more the graph is expanded and the slower the algorithm performs. Additionally, the location of the start and goal region has an effect on the runtime. If the start area is situated in a region of a major fiber bundle where anisotropy and therefore the walking direction are well defined, the algorithm has to spread out less and performs faster. Contrarily, if the start region is situated near the cortex, the tensor information is more diffuse and the pathfinding process has to spread out to a greater search area. This also applies to goal areas that are hard to reach, either if they are located at areas with low anisotropy or if the algorithm has to cross such regions.

The adjustment of cost functions is also an important aspect which influences the runtime of the approach as well as the quality of fiber connection. In general, it has been proven that the probability derived from the tensor shape should obtain a high influence on the cost function. The bending component prevents the algorithm from twisting and spreading out unnecessarily in areas where the diffusion direction is less apparent. The walkability threshold as well as the cost function component based on FA help the algorithm to constrain the search area and to proceed faster. However, if the start or goal region are located in areas of weak anisotropy, these thresholds must be lowered resulting in a higher computational expense. 

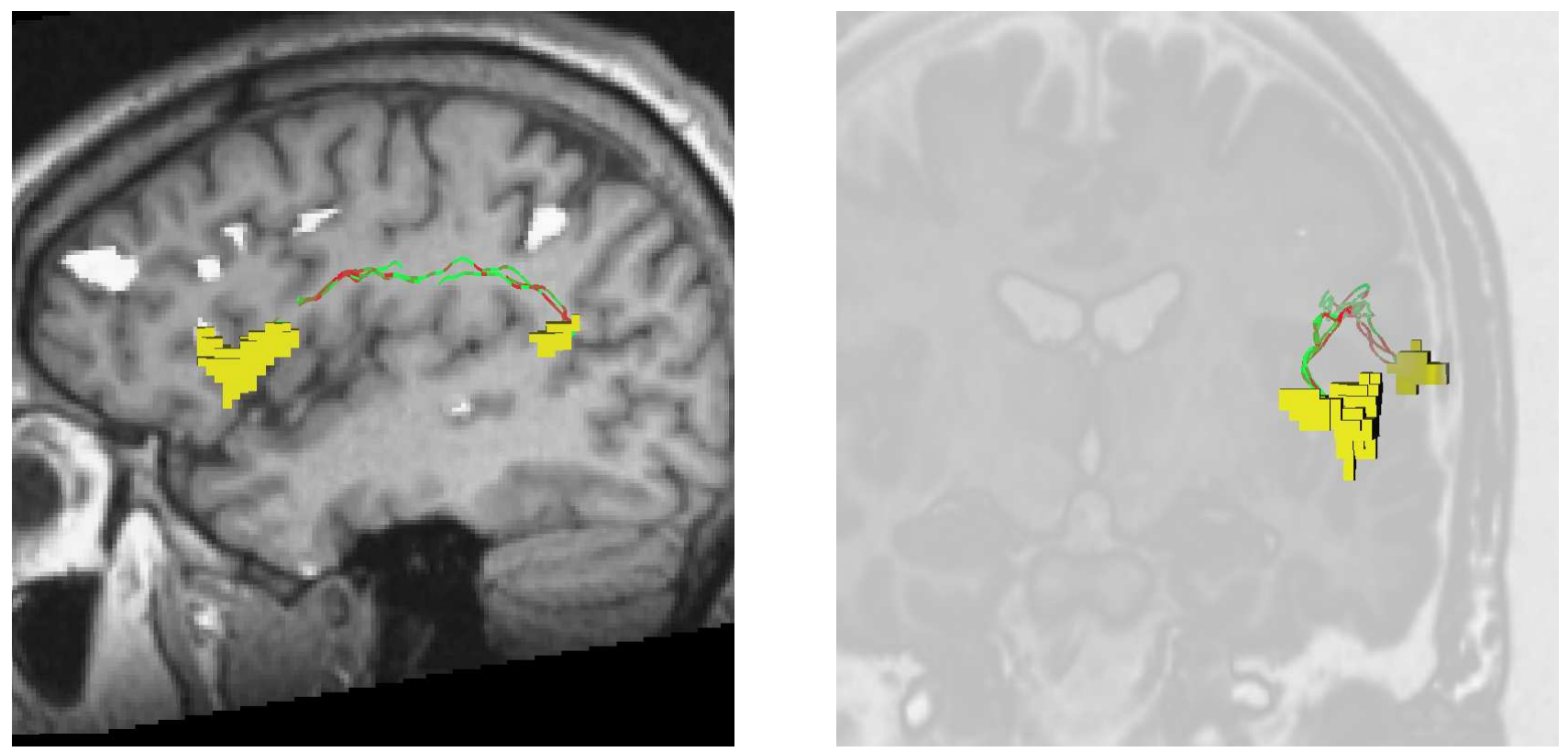

Figure 4. Connection computed between speech areas based on fMRI data displayed with anatomical data. The resulting connection shows an arc shape which is conform to medical knowledge.

\section{CONCLUSION AND FUTURE WORK}

A novel approach for establishing a connection between areas within the brain using pathfinding was proposed. Appropriate cost functions based on the probability distribution function of the tensor are incorporated guiding the pathfinding procedure. The approach is suited to extract authentic pathways and to provide probability information for each single path segment. From the derived brain connectivity information a better understanding of brain function and structure is anticipated.

Future work will focus on further evaluation of this approach for surgical application. Identified fiber connections relating motor and sensory speech areas are of high importance especially in patients with adjacent lesions to prevent neurological deficits. Incorporation of these techniques into surgical planning will therefore be of high value.

\section{ACKNOWLEDGMENTS}

This work was supported by the Deutsche Forschungsgemeinschaft in the context of SFB 603, Project C9 and the Graduate Research Center "3D Image Analysis and Synthesis". We are especially grateful to Peter Grummich (Dept. of Neurosurgery, University of Erlangen-Nuremberg, Germany) for speech fMRI.

\section{REFERENCES}

1. L. Zhukov and A. Barr, "Oriented Tensor Reconstruction: Tracing Neural Pathways from Diffusion Tensor MRI," in Proc. IEEE Visualization, 2002.

2. C. Poupon, C. A. Clark, V. Frouin, J. Régis, I. Bloch, D. L. Bihan, and J.-F. Mangin, "Regularization of diffusion-based direction maps for the tracking of brain white matter fascicles," Neuroimage 12(2), pp. 184$195,2000$.

3. T. Behrens, H. Johansen-Berg, M. Woolrich, S. Smith, C. Wheeler-Kingshott, P. Boulby, G. Barker, E. Sillery, K. Sheehan, O. Ciccarelli, A. Thompson, J. Brady, and P. Matthews, "Non-invasive mapping of connections between human thalamus and cortex using diffusion imaging," Nature Neuroscience 6(7), pp. $750-757,2003$. 
4. M. Björnemo, A. Brun, R. Kikinis, and C.-F. Westin, "Regularized stochastic white matter tractography using diffusion tensor MRI," in Proc. MICCAI, 2002.

5. P. Batchelor, D. Hill, F. Calamante, and D. Atkinson, "Study of connectivity in the brain using the full diffusion tensor from MRI," in Information Processing in Medical Imaging, 17th International Conference, pp. 121-133, 2001.

6. L. O'Donnell, S. Haker, and C.-F. Westin, "New approaches to estimation of white matter connectivity in diffusion tensor MRI: Elliptic PDEs and geodesics in a tensor-warped space," in Proc. MICCAI, pp. 459-466, 2002.

7. R. Henry, J. Berman, S. Nagarajan, P. Mukherjee, and M. Berger, "Subcortical pathways serving cortical language sites: initial experience with diffusion tensor imaging fiber tracking combined with intraoperative language mapping," Neuroimage 21(2), pp. 616-622, 2004.

8. J.-C. Latombe, Robot Motion Planning, Kluwer Academic Publishers, 1991.

9. P. E. Hart, N. J. Nilsson, and B. Raphael, "A formal basis for the heuristic determination of minimum cost paths," in Autonomous Mobile Robots: Perception, Mapping, and Navigation, S. S. Iyengar and A. Elfes, eds., pp. 375-382, IEEE Computer Society Press, 1991.

10. N. Nilsson, Principles of Artificial Intelligence, Tioga Publishing Company, 1980.

11. R. Dechter and J. Pearl, "Generalized best-first search strategies and the optimality of A*," Journal of the ACM 32(3), pp. 505-536, 1985. 